\title{
MOBILIDADE NA MARCHA, RISCO DE QUEDAS E DEPRESSÃO EM IDOSOS INSTITUCIONALIZADOS E NÃO INSTITUCIONALIZADOS
}

\section{Camila Costa}

Graduada em Fisioterapia (FAA), Brasil.

Carla Gabriela Kemer

Graduada em Fisioterapia (FAA), Brasil.

\section{Daniel Vicentini Oliveira}

Doutorando em Gerontologia (UNICAMP); Docente no Departamento de Educação física da Faculdade Metropolitana de Maringá (FAMMA), Brasil.

\section{Mateus Dias Antunes}

Mestrando em Promoção da Saúde (UNICESUMAR), Brasil.

José Roberto Andrade do Nascimento Júnior

Doutor em Educação física (UEM); Docente no Departamento de Educação física da UNIVASF, Brasil.

Cristina Cristovão Ribeiro da Silva

Doutoranda em Gerontologia (UNICAMP), Brasil.
RESUMO: Este artigo tem como objetivo comparar o risco de quedas, indicativos de depressão e mobilidade na marcha de idosos institucionalizados e idosos frequentadores de um centro de convivência do município de Foz do Iguaçu, estado do Paraná. Trata-se de um estudo transversal, constituído por 26 idosos de ambos os sexos, sendo 13 institucionalizados (ILPI) e 13 frequentadores de um centro de convivência (CCI). Foi utilizado um questionário sociodemográfico, a Escala de Equilíbrio de Berg, escala de depressão geriátrica e o teste Timed Up and go. A análise dos dados foi realizada mediante os testes Shapiro-Wilk, "U" de Mann-Whitney, a correlação de Spearman e o teste Qui-quadrado e o teste exato de Fisher. Foi adotada a significância de $\mathrm{p}<0,05$. Ressalta-se que, significativamente, idosos do CCI apresentaram menor indicativo de depressão $(p=0,009)$, menor risco de quedas $(\mathrm{p}=0,001)$ e melhor mobilidade na marcha $(\mathrm{p}=$ 0,001 ) em detrimento dos idosos do ILPI. Após a identificação de que os idosos institucionalizados apresentam maior risco de quedas, dificuldade na marcha e de sinais depressivos quando comparados aos não institucionalizados, surge um desafio para os serviços de saúde no enfrentamento da incapacidade funcional ao longo da vida.

PALAVRAS-CHAVE: Envelhecimento; Gerontologia; Atividade Motora, Promoção da Saúde.

\section{MOBILIT Y IN GAIT, RISK OF FALLS AND DEPRESSION IN INSTITUTIONALIZED AND NON- INSTITUTIONALIZED ELDERLY}

ABSTRACT: This paper aims to compare the risk of falls, indicatives of depression and mobility in the gait of institutionalized elderly and elderly people attending a community center in the city of Foz do Iguaçu, Paraná state. It is a cross-sectional study with 26 elderly of both sexes, 13 institutionalized (ILPI) and 13 attending a community center (CCI). A sociodemographic questionnaire, Berg Balance Scale, Geriatric Depression Scale and the Timed Up and Go Test were used. Data analysis was performed using the Shapiro-Wilk, Mann-Whitney "U" tests, Spearman correlation, and the Chi-square and Fisher's exact test. The significance of $p<0.05$ was adopted. It is emphasized that, significantly, the elderly of CCI had a lower rate of depression $(\mathrm{p}=$ $0.009)$, a lower risk of falls $(p=0.001)$ and a better gait mobility ( $p$ $=0.001$ ), in contrast to the elderly of the ILPI. After identification that the institutionalized elderly present a higher risk of falls and gait difficulties, as well as depressive signs when compared to noninstitutionalized ones, a challenge arises for the health services when facing the functional disability throughout life.

KEY WORDS: Aging; Gerontology; Motor Activity, Health Promotion. 


\section{INTRODUÇÃO}

A mudança epidemiológica ocorreu quando processos agudos eram curados ou as pessoas iam a óbito. Com o avanço da saúde e da tecnologia de diagnóstico, as doenças crônicas prevalecem e as complicações geram incapacidades e dependência, aumentando a demanda dos serviços de saúde. O grande desafio será conceder aos idosos melhoria na qualidade de vida e prolongar sua autonomia e independência funcional nas atividades de vida diária (AVDs) (RIBEIRO, 2012).

No cotidiano dos idosos, diversos fatores facilitam ou propiciam a ocorrência de quedas. Esses fatores podem ser intrínsecos, inerentes ao próprio indivíduo, relacionados às alterações biológicas e psicossociais associadas a senescências. Já os fatores extrínsecos são decorrentes da interação do idoso com o ambiente, como a qualidade da iluminação e do piso de sua moradia, o acesso a transportes públicos e áreas de lazer, entre outros. Nota-se que esses fatores podem ser multifatoriais e apresentam relação com a capacidade de manter habilidades para realizar as atividades do dia a dia (SMITH et al., 2017).

A capacidade funcional é definida como o potencial que os idosos apresentam para decidir e atuar em suas vidas de forma independente, no se cotidiano. Já a incapacidade funcional é a necessidade de ajuda ou dificuldade em executar as tarefas do seu dia-a-dia. Nesse sentido, evidencia-se que a incapacidade funcional atua no aumento do risco de quedas, bem como na prevalência de situações que promovem o estresse na terceira idade devido às limitações impostas (BARBOSA et al., 2014).

A restrição às atividades, o isolamento social, o declínio da saúde, a institucionalização e o aumento do índice de depressão são consequências do risco de quedas. Com o avanço da idade surgem alterações psicológicas, há mudança na rotina e dos problemas a serem enfrentados, o que predispõe à depressão. O quadro depressivo no idoso é responsável pelo isolamento social e agravo do declínio funcional (SILVA et al., 2017).

$O$ processo de institucionalização gera sentimento de sofrimento pela separação da família e abandono, perda de liberdade e percebe-se, com maior intensidade, a aproximação da morte (RALDI; CANTELE; PALMEIRAS, 2017). A perda de uma vida independente é uma das razões para a institucionalização, onde o idoso deverá encontrar os cuidados necessários para manter e aumentar sua funcionalidade. Com a imperícia da família, acentua-se a procura por ILPIs (ANDRADEA et al., 2014; ZAGONEL et al., 2017).

Ao realizar uma tarefa da vida diária é preciso a união das funções cognitivas, motoras e psicológicas. Seu detrimento poderá ocasionar dependência e incapacidades. Diante disso, a avaliação da capacidade funcional é essencial para a escolha do melhor tipo de intervenção. A avaliação consiste em mensurar em que nível o idoso encontra-se em várias áreas, como a integridade física, qualidade da automanutenção, estado intelectual, atividades sociais, estado emocional e autocuidado. Após este diagnóstico, os dados podem colaborar para estabelecer um plano de tratamento adequado às demandas identificadas (COSTA et al., 2017) .

Este artigo teve como objetivo comparar o risco de quedas, indicativos de depressão e mobilidade na marcha de idosos institucionalizados e idosos frequentadores de um centro de convivência do município de Foz do Iguaçu, estado do Paraná.

\section{METODOLOGIA}

Trata-se de um estudo quantitativo, transversal e observacional, realizado em Foz do Iguaçu, Paraná, com idosos independentes institucionalizados (ILPI) e não institucionalizados frequentadores de um centro de conveniência para idosos (CCI).

Foram incluídos idosos independentes nas atividades básicas de vida diária (ABVDs) sem comprometimento cognitivo. Esses últimos foram avaliados por meio do mini-exame do estado mental (MEEM), que fornece dados sobre diferentes parâmetros cognitivos. Ele examina a orientação temporal e espacial, a memória de curto prazo, o cálculo, a praxia e a linguagem. Sua pontuação máxima é de 30 pontos e os idosos são classificados de acordo com a escolaridade, de modo que foram atribuídos 18 pontos para idosos analfabetos, 21 para idosos com 1 a 3 anos de escolaridade, 24 para 4 a 7 anos de escolaridade, 26 para 8 ou mais anos de escolaridade (AVEIRO et al., 2012). Idosos que obtiveram escore igual ou superior aos relatados também foram incluídos.

O Índice de Katz foi utilizado para verificar a dependência funcional do idoso nas ABVDs. O mesmo avalia a capacidade do idoso na alimentação, banho, 
vestuário, transferência da cama para a cadeira e viceversa, locomoção ao sanitário e continência (POLARO et al., 2012). Foram considerados independentes os idosos que informaram não precisar de ajuda em nenhuma dessas atividades. Os critérios de exclusão foram idosos com comprometimento cognitivo e dependência parcial ou total nas ABVDs.

Foi utilizado um questionário com dados sociodemográficos, semiestruturado pelos próprios autores, com perguntas referentes a sexo (masculino ou feminino), faixa etária (60 a 69 anos, 70 anos ou mais), tem filhos (sim ou não), hospitalização no último semestre (sim ou não), uso de medicamentos (sim ou não), presença de doenças crônicas (sim ou não), percepção de saúde (boa, regular ou ruim), histórico de quedas no último semestre (sim ou não), uso de utensílio para marcha (sim ou não).

A escala de equilíbrio de Berg (balance scale) é constituída por 14 tarefas funcionais que envolvem equilíbrio corporal estático e dinâmico, tais como alcançar, girar, transferir-se, permanecer em pé e levantarse. Ela é avaliada através da observação e sua pontuação varia de 0 a 4 , totalizando o 56 pontos. Quanto menor a pontuação, maior é o risco de quedas (MEMÓRIA et al., 2016).

A escala de depressão geriátrica (GDS) é um teste para detectar sintomas depressivos no idoso. Composta por 15 perguntas com sim e não como opção de resposta, tem score total que varia de 0 a 15 pontos. Entre 0 e 5 pontos, o idoso está dentro da normalidade, 6 a 10 pontos indica possível depressão e 11 a 15 pontos indica possível depressão grave (PEREIRA et al., 2014; ALMEIDA; ALMEIDA,1999).

$\mathrm{O}$ teste Timed Up and Go (TUG) quantifica em segundos a mobilidade através da velocidade do idoso em levantar-se de uma cadeira sem ajuda dos braços, andar uma distância de três metros, dar a volta e retornar. A velocidade atingida está relacionada com o risco de quedas. Assim, menos de 10 segundos sugere indivíduos livres e independentes, entre 10 e 19 segundos, eles são independentes, pois têm razoável equilíbrio e velocidade de marcha, 20 e 29 segundos significa idosos com dificuldades variadas para as tarefas da vida diária, dependendo das diferentes situações que se apresentam ao indivíduo, 30 segundos ou mais indica idosos que tendem a ser totalmente dependentes para muitas
ABVDS e atividades instrumentais da vida diária (AIVDs) (BRETAN et al., 2013).

A coleta de dados foi feita entre maio e agosto de 2016. A princípio, foi requisitada a autorização das instituições. Após a aprovação pelo Comitê de Ética e Pesquisa (Parecer $\left.n^{0} 1.501 .410\right)$, deu-se início à coleta de dados. Os testes aplicados foram realizados em conjunto pelas pesquisadoras para que não houvesse divergência de resultados. Todos os participantes assinaram o Termo de Consentimento Livre e Esclarecido.

Os dados foram analisados no software SPSS versão 22.0. Foi utilizada estatística descritiva e inferencial. Para as variáveis categóricas utilizou-se frequência e percentual. Já para as variáveis numéricas, inicialmente verificou-se a normalidade dos dados por meio do teste de Shapiro-Wilk. Foi usada Mediana/Quartis (dados não normais) para a caracterização dos resultados. Para a comparação da funcionalidade entre os grupos ILPI e CCI, foi utilizado o teste "U" de Mann-Whitney. Foi utilizado o coeficiente de correlação de Spearman para verificar a correlação entre a depressão, risco de quedas e o TUG. As possíveis comparações das proporções de idosos dos grupos ILPI e CCI em função das variáveis sociodemográficas e de funcionalidade foram analisadas por meio do teste qui-quadrado ou o teste exato de Fisher. Foi adotada a significância de $p<0,05$.

\section{RESULTADOS}

Fizeram parte deste estudo 26 idosos independentes, sendo 13 alocados na ILPI com média de idade de 71,8 \pm 6,2anos (10 homens e 3 mulheres) e 13 alocados no CCI, com média de idade de 67,7 $\pm 5,6$ anos (10 homens e 3 mulheres).

Ao comparar as proporções de idosos entre os grupos (ILPI e CCI) em função das variáveis sociodemográficas e de saúde (Tabela 1), foi encontrada diferença significativa no número de filhos $(\mathrm{p}=0,039)$, uso de medicamentos $(\mathrm{p}=0,001)$, indicativo de depressão $(\mathrm{p}=0,016)$ e risco de quedas ( $\mathrm{p}=0,007)$. Ressalta-se que existe uma maior proporção de indivíduos do grupo CCI que têm filhos ( $f=11$ ), não usam medicamentos ( $f$ $=11)$, não apresentam indicativos de depressão $(\mathrm{f}=11)$ e têm baixo risco de quedas ( $\mathrm{f}=13$ ). 
Tabela 1. Comparação das proporções de idosos entre os grupos ILPI e CCI em função das variáveis sociodemográficas e de saúde

\begin{tabular}{|c|c|c|c|c|}
\hline \multirow{3}{*}{ VARIÁVEIS } & \multicolumn{2}{|c|}{ Grupos } & \multirow{3}{*}{$\mathbf{X}^{2}$} & \multirow{3}{*}{$\mathbf{P}$} \\
\hline & ILPI & CCI & & \\
\hline & $f(\%)$ & $f(\%)$ & & \\
\hline \multicolumn{5}{|l|}{$\operatorname{Sexo}^{\mathrm{a}}$} \\
\hline Masculino & $3(50,0)$ & $3(50,0)$ & \multirow{2}{*}{0,000} & \multirow{2}{*}{1,000} \\
\hline Feminino & $10(50,0)$ & $3(50,0)$ & & \\
\hline \multicolumn{5}{|l|}{ Faixa etária $^{a}$} \\
\hline 60 a 69 anos & $5(35,7)$ & $9(64,3)$ & \multirow{2}{*}{2,476} & \multirow{2}{*}{0,116} \\
\hline 70 anos ou mais anos & $8(66,7)$ & $4(33,3)$ & & \\
\hline \multicolumn{5}{|l|}{ Filhos $^{b}$} \\
\hline Sim & $6(35,3)$ & $11(64,7)$ & \multirow{2}{*}{4,248} & \multirow{2}{*}{$0,039 *$} \\
\hline Não & $7(77,8)$ & $2(22,2)$ & & \\
\hline \multicolumn{5}{|l|}{ Hospitalização $^{\text {b }}$} \\
\hline Sim & $5(83,3)$ & $1(16,7)$ & \multirow{2}{*}{3,467} & \multirow{2}{*}{0,080} \\
\hline Não & $8(40,0)$ & $12(60,0)$ & & \\
\hline \multicolumn{5}{|l|}{ Medicamentos $^{\mathrm{a}}$} \\
\hline Sim & $11(84,6)$ & $2(15,4)$ & \multirow{2}{*}{12,462} & \multirow{2}{*}{$0,001^{*}$} \\
\hline Não & $2(15,4)$ & $11(84,6)$ & & \\
\hline \multicolumn{5}{|l|}{ Doenças crônicas $^{\text {b }}$} \\
\hline Sim & $7(41,2)$ & $10(58,8)$ & \multirow{2}{*}{1,529} & \multirow{2}{*}{0,205} \\
\hline Não & $6(66,7)$ & $3(33,3)$ & & \\
\hline \multicolumn{5}{|l|}{ Percepção de saúde ${ }^{\text {b }}$} \\
\hline Boa & $9(45,0)$ & $11(55,0)$ & \multirow{2}{*}{0,867} & \multirow{2}{*}{0,352} \\
\hline Regular & $4(66,7)$ & $2(33,3)$ & & \\
\hline \multicolumn{5}{|l|}{ Histórico de quedas ${ }^{\mathrm{b}}$} \\
\hline Sim & $3(60,0)$ & $2(40,0)$ & \multirow{2}{*}{0,248} & \multirow{2}{*}{0,500} \\
\hline Não & $10(47,6)$ & $11(52,4)$ & & \\
\hline \multicolumn{5}{|c|}{ Utiliza dispositivo para marcha ${ }^{\mathrm{b}}$} \\
\hline Sim & $1(100,0)$ & $0(0,0)$ & 1040 & 0500 \\
\hline Não & $12(48,0)$ & $13(52,0)$ & 1,010 & 0 \\
\hline Indicativo de depressão ${ }^{a}$ & & & & \\
\hline Sim & $8(80,0)$ & $2(20,0)$ & 5850 & 0.016* \\
\hline Não & $5(31,2)$ & $11(68,8)$ & & \\
\hline Risco de quedas ${ }^{b}$ & & & & \\
\hline Baixo risco & $7(35,0)$ & $13(65,0)$ & 7800 & $0.007 *$ \\
\hline Moderado/alto risco & $6(100,0)$ & $0(0,0)$ &, 000 & ( \\
\hline
\end{tabular}

*Associação significativa - p < 0,05: a) Teste de Qui-quadrado; b) Teste Exato de Fisher. Fonte: Dados da pesquisa 
Ao comparar o indicativo de depressão, o risco de quedas e o teste de mobilidade (TUG) dos idosos dos grupos ILPI e CCI (Tabela 2), verificou-se diferença significativa em todas as variáveis $(\mathrm{p}<0,05)$. Destacase que os idosos do grupo CCI apresentaram menor indicativo de depressão $(\mathrm{Md}=2,0)$, menor risco de quedas (Md =54,0) e melhor mobilidade na marcha (Md $=9,0)$ em detrimento aos idosos do grupo ILPI $(\mathrm{Md}=$ 5,$0 ; \mathrm{Md}=41,0 ; \mathrm{Md}=16,0$, respectivamente).

Tabela 2. Comparação do indicativo de depressão, risco de quedas e teste de mobilidade (TUG) dos idosos dos grupos ILPI e CCI

\begin{tabular}{|c|c|c|c|}
\hline \multirow{2}{*}{ VARIÁVEIS } & ILPI & CCI & \multirow{2}{*}{$P$} \\
\hline & Md (Q1;Q3) & Md (Q1;Q3) & \\
\hline $\begin{array}{l}\text { Indicativo de } \\
\text { depressão }\end{array}$ & $5,0(3,0 ; 7,0)$ & $2,0(0,0 ; 3,5)$ & $0,009 *$ \\
\hline Risco de quedas & $\begin{array}{c}41,0(37,0 \\
47,5)\end{array}$ & $\begin{array}{c}54,0(52,5 \\
56,0)\end{array}$ & $0,001 *$ \\
\hline $\begin{array}{l}\text { Teste de mobilidade } \\
\text { (TUG) }\end{array}$ & $\begin{array}{l}16,0(13,0 \\
24,0)\end{array}$ & $9,0(8,0 ; 11,0)$ & $0,001^{*}$ \\
\hline
\end{tabular}

* Diferença significativa: $\mathrm{p}<0,05$ - Teste "U" de Mann-Whitney. Fonte: Dados da pesquisa

Ao analisar a correlação entre o indicativo de depressão, o risco de quedas e a mobilidade para a marcha dos idosos do grupo ILP e CCII, observou-se correlação significativa $(\mathrm{p}<0,05)$ apenas entre a mobilidade para a marcha (TUG) e o risco de quedas. Nos idosos do grupo ILPI foi encontrada correlação forte $(r=-0,86)$ entre as variáveis, indicando uma relação grande e inversamente proporcional, isto é, quanto menor o risco de quedas, maior a mobilidade para a marcha e vice-versa. Já entre os idosos do grupo CCI foi encontrada correlação moderada e inversamente proporcional entre a mobilidade para a marcha (TUG) e o risco de quedas $(r=-0,54)$.

\section{DISCUSSÃO}

Verificou-sequetantoosidososinstitucionalizados quanto os não institucionalizados têm uma boa percepção da saúde. Mesmo quando apontam o uso de medicamentos e a existência de alguma doença crônica associada, eles não se consideram doentes. Este dado está de acordo com a pesquisa realizado por Oliveira, Pedrosa e Santos (2009) na cidade do Sertão Pernambucano, com 100 idosos de 60 a 97 anos, onde todos tomavam medicação em decorrência de alguma complicação de saúde, porém nem por isso consideravam-se doentes. A explicação para tal resultado é que a saúde percebida pelo idoso está relacionada com a incapacidade e não com serem portadores de doença crônica. Portanto, quanto maior o grau de dependência, maior será a autopercepção como ruim (MEDEIROS et al., 2016).

Quando analisa-se os idosos que sofreram quedas nos últimos 12 meses, nota-se que eram solteiros e tomavam medicamentos. Tal achado mostra que os idosos separados, viúvos ou divorciados apresentam aumento no risco de queda. Os idosos que vivem com companheiro demonstram menor risco de quedas, sendo elucidado pelo cuidado recíproco entre ambos. Resultados similares foram encontrados em dois estudos, nos quais cerca de $28,5 \%$ já caíram mais de uma vez e não têm vida conjugal. Esta prevalência de quedas sobe para $32,5 \%$ entre os idosos sem vida conjugal e que moram só, e para 39,0\% entre os idosos sem vida conjugal que moram em domicílios de uma geração (PERRACINI, RAMOS, 2002).

Outro estudo, ao analisar a prevalência de quedas em idosos e a influência de variáveis a elas associadas, mostrou que as quedas foram mais frequentes no sexo feminino $(p<0,001)$, nos mais velhos $(p<0,001)$, nos idosos separados, divorciados e viúvos $(p<0,001)$ e nos do nível socioeconômico mais baixo $(p=0,04)$. O percentual de quedas também esteve associado ao sedentarismo, autopercepção de saúde ruim e maior número de medicamentos referidos para uso contínuo (SIRQUEIRA et al., 2007).

A queda é um evento grande e preocupante entre os idosos, causando alterações físicas e psicológicas. Em sua grande maioria, as quedas ocorrem no local de moradia. Estudos apontam que idoso que vive em ILPIs sofrem maior número de quedas do que aqueles que vivem na comunidade em razão das poucas atividades físicas oferecidas, agravando o ciclo envelhecimento, menor capacidade funcional e sedentarismo (SOUZA et al., 2013; MATHEW; VANLALPEKI; NAIR, 2017). 
Corroborando com o presente estudo segundo o qual os idosos institucionalizados caem mais do que os não institucionalizados, isto é, 23\% dos idosos institucionalizados e $15 \%$ dos idosos não institucionalizados relataram ter sofrido queda.

Todavia, a avaliação do equilíbrio corporal estático e dinâmico é de extrema importância para identificar o risco de cair. Desse modo, no presente estudo foi aplicada a escala de Berg, resultando em uma pontuação de 40,30 para idosos institucionalizados e 53,84 pontos para os idosos não institucionalizados, o que indica o risco de quedas nos idosos institucionalizados. Outro teste realizado foi Time Up and Go (TUG). Os resultados obtidos constataram comprometimento funcional entre os idosos institucionalizados, que realizaram o teste em tempo maior que 20 segundos.

A senescência associada a fatores extrínsecos e a por processos patológicos torna o idoso vulnerável a quedas. Fraturas, medo de cair, perda da mobilidade, ansiedade, depressão e, em alguns casos, a morte são consequência desse evento. A prática regular de atividade física pode amenizar o processo de envelhecimento e prevenir o sedentarismo, contribuindo para exercer atividades do dia a dia, aumentando a expectativa de vida funcional, visando à diminuição das quedas, com ênfase nos exercícios de movimentação articular livre, fortalecimento global, treinamento de marcha, equilíbrio, propriocepção e treinamento funcional (KING; KING, 2017).

O desempenho cognitivo avaliado através no MEEM constatou que 5 dos 13 idosos institucionalizados apresentaram déficit cognitivo, ficando abaixo do ponto de corte para sua escolaridade. Esses dados assemelhamse ao estudo realizado por Bertoldi, Batista e Ruzanowsky (2015), que se refere a uma revisão de literatura onde o objetivo era investigar a presença de déficit cognitivo em pacientes institucionalizados. Em uma das pesquisas, 22 idosos institucionalizados e 31 idosos não institucionalizados, com faixa etária de $72,33 \pm 10$ e ambos os sexos, foram avaliados através do MEEM, que identificou comprometimento cognitivo severo no primeiro grupo, com resultado de 11,73 $\pm 6,04$, e funções preservadas no segundo grupo, com média de $26,39 \pm 3,2.11$.
Em outro estudo, observou-se que $60 \%$ de 60 idosos institucionalizados, com idade de 75,0 $\pm 11,60$ anos, tem alteração cognitiva. O autor cita que a institucionalização é um fator para o declínio das funções mentais, pois o idoso deixa para trás sua identidade, seus objetos, sua residência e lembranças de uma vida inteira que não voltam mais. A partir dessa nova fase, ele terá que construir um novo significado para a vida, com regras, normas, horários e novos relacionamentos, levando à perda de liberdade, autoestima e ao estado de solidão. Isso pode desencadear a depressão, sendo um fator de risco para o déficit cognitivo e demência.

Contudo, faz-se necessário detectar sintomas depressivos no idoso. Assim, em nossa pesquisa os idosos foram avaliados através da escala de depressão geriátrica (GDS), sendo que 5 dos 13 idosos institucionalizados apresentaram sugestão de depressão. Portanto, é imprescindível a criação e manutenção de alternativas que visem a autonomia do idoso, atividades expressivas que não só preencham o tempo ocioso, mas que dêem sentido ao seu cotidiano (FLORES; DEODATO; MATTION, 2016).

É de grande importância a prática de atividades físicas no processo de envelhecimento, inclusive técnicas de revitalização geriátrica e da utilização da fisioterapia na prevenção e promoção na saúde do idoso. São inúmeros os benefícios, entre eles a prevenção de síndrome do aparelho respiratório, prevenção de alterações posturais, perda da força e preensão bilateral das mãos, melhoria da flexibilidade corporal, facilitando a realização das AVDs e AIVDs, tendo como consequência uma melhoria na qualidade de vida e satisfação pessoal (MENEZES et al., 2016).

Ao realizar uma tarefa da vida diária é preciso a união das funções cognitivas, motoras e psicológicas. Seu detrimento poderá causar dependência e incapacidades. Em função disso, avaliar a capacidade funcional é essencial para a escolha do melhor tipo de intervenção e para mensurar em que nível o idoso enquadra-se em várias áreas, como a integridade física, qualidade da automanutenção, estado intelectual, atividades sociais, estado emocional e cuidados a si mesmo. A consequência é o estabelecimento de um plano de tratamento adequado às demandas identificadas (COSTA et al., 2017). 
A partir daí, sugere-se que novas pesquisas sejam realizadas nesta área com protocolos de avaliação que permitam comparar mais dados, como a baropodomotria, para análise do equilíbrio e câmeras 3D, e a plataforma de força, para analisar a biomecânica do movimento e o risco de quedas. Espera-se que este estudo possa contribuir para pesquisas futuras sobre a funcionalidade da população idosa.

\section{CONCLUSÃO}

Conclui-se que idosos não institucionalizados têm menor risco de quedas, menores indicativos de depressão e melhor mobilidade na marcha do que idosos institucionalizados. A magnitude e severidade dos achados, neste estudo, constituem um desafio para os serviços de saúde no enfrentamento da incapacidade funcional ao longo da vida. Nesse sentido, busca-se contribuir para direcionar o planejamento de práticas que promovem a saúde dos idosos institucionalizados.

\section{REFERÊNCIA}

ALMEIDA, O.P.; ALMEIDA, A.S. Confiabilidade da versão brasileira da Escala de Depressão Geriátrica (GDS) versão reduzida. Arquivos de Neuropsiquiatria, v. 57, n. 2, p. 421-426, 1999.

ANDRADEA, N.B. et al. Centro de convivência de idosos: uma abordagem de estimulação cognitiva e psicossocial. Cadernos de Terapia Ocupacional da USP, v. 22, n. 1, p. 121-128, 2014.

AVEIRO, M.C. et al. Mobilidade e risco de quedas de população idosa da comunidade de São Carlos. Ciência \& Saúde Coletiva, v. 17, n. 9, p. 2481-2488, 2012.

AZEVEDO, C. Tecnologias e pessoas mais velhas: as novas tecnologias de informação e comunicação nas relações sociais de seniores em Portugal. Revista Portuguesa de Ciências da Comunicação, v. 1, n. 1, p. 1171-1175, 2016.

BARBOSA, B. R. et al. Avaliação da capacidade funcional dos idosos e fatores associados à incapacidade. Ciência \&
Saúde Coletiva, v. 19, n. 8, p. 3317-3325, 2014.

BERTOLDI, J.T.; BATISTA, A.C.; RUZANOWSKY, S. Declínio cognitivo em idosos institucionalizados: revisão de literatura. Cinergis, v. 16, n. 2, p.152-156, 2015.

BRETAN, O. et al. Risco de queda em idosos da comunidade: avaliação com o teste Timed up and go. Brazilian Journal of Otorhinolaryngol, v. 79, n 1, p. 18-21, 2013.

COSTA, K.M.S.M. et al. Perfil antropométrico, funcional e cognitivo de idosos não institucionalizados. Revista Brasileira de Pesquisa em Ciências da Saúde, v. 3, n. 2, p. 28-35, 2017.

FLORES, G.C.; DEODATO, S.; MATTIONI, F.C. A relação entre autonomia e a condição de ser idoso na literatura científica de enfermagem: uma revisão integrativa. Revista de Extensão da Univasf, v. 4, n. 1, p. 139-150, 2016.

KING, A.; KING, D. Physical activity for an aging population. Public Health Reviews, v. 32, n. 2, p. 401426, 2017.

MATHEW, J.; VANLALPEKI, T.; NAIR, G. G. Gait Evaluation of Institutionalized Elders-A Feasibility Study. Indian Journal of Gerontology, v. 31, n. 1, p. 71-83, 2017.

MEDEIROS, S.M. et al. Fatores associados à autopercepção negativa da saúde entre idosos não institucionalizados de Montes Claros, Brasil. Ciência \& Saúde Coletiva, v. 21, n. 11, p. 3377-3386, 2016.

MEMÓRIA, A.K.U.B. et al. Uso de instrumentos para a investigação do equilíbrio postural em tarefas funcionais. Fisioterapia Brasil, v. 17, n. 6, p. 585-595, 2016.

MENEZES, A.V. et al. Função executiva de idosos institucionalizados e comunitários: relação com capacidades cognitivas e funcionais. Saúde e Pesquisa, v. 9 , n. 3, p. 405-414, 2016.

OLIVEIRA, S.C.F.; PEDROSA, M.I.P.C.; SANTOS, M.F.S. Doença versus saúde: como os idosos se percebem diante desses conceitos. Revista Brasileira de Ciências do Envelhecimento Humano, v. 6, n. 2, p. 181-188, 2009.

PEREIRA, E.E.B. et al. Funcionalidade global de idosos hospitalizados. Revista Brasileira de Geriatria e 
Gerontologia, v. 17, n. 1, p. 165-176, 2014.

PERRACINI, R.P.; RAMOS, L.R. Fatores associados a quedas em uma coorte de idosos residentes na comunidade. Revista de Saúde Pública, v. 36, n. 6, p. 709-716, 2012.

POLARO, S.H.I. et al. Idosos residentes em instituições de longa permanência para idosos da região metropolitana de Belém-PA. Revista Brasileira de Geriatria e Gerontologia, v. 15, n. 4, p. 777-784, 2012.

RALDI, G. V.; CANTELE, A. B.; PALMEIRAS, G. B. Avaliação da prevalência de depressão em idosos institucionalizados em uma ILPI no norte do RS. Revista de Enfermagem, v. 12, n. 12, p. 48-63, 2017.

RIBEIRO, C. Envelhecimento e o Paradigma Funcional. In: RIBEIRO, Cristina. As 8 Premissas da Fisioterapia Gerontológica: A atuação fisioterapêutica sob a ótica da gerontologia. São Paulo: Andrei. 2012. cap.1, p. 23-34.

SILVA, J. C. A. et al. Associação entre o risco de queda e o índice de depressão em idosos. SANARE-Revista de Políticas Públicas, v. 15, n. 2, p. 8-14, 2017.

SIQUEIRA, F.V et al. Prevalência de quedas em idosos e fatores associados. Revista de Saúde Pública, v. 41, n. 5 , p. 749-756, 2007.

SMITH, A. A. et al. Avaliação do risco de quedas em idosos residentes em domicílio. Revista Latino-Americana de Enfermagem, v. 25, p. 1-9, 2017.

SOUZA, C.C. et al . Mobilidade funcional em idosos institucionalizados e não institucionalizados. Revista Brasileira de Geriatria e Gerontologia, v. 16, n. 2, p. 285-293, 2013.

ZAGONEL, A.D. et al. As percepções sociais frente a implantação de uma Instituição de Longa Permanência para Idosos em um município do Vale do Taquari/RS, Brasil. Scientia Plena, v. 13, n. 2, p. 1-7, 2017.

Recebido em: 28 de maio de 2017 Aceito em: 10 de agosto de 2017 